\title{
Challenges to the Structural Conception of Chemical Bonding*
}

\author{
Michael Weisberg \\ University of Pennsylvania \\ forthcoming in Philosophy of Science
}

\begin{abstract}
While the covalent bond plays a central role in chemical predictions, interventions, and explanations, it is a difficult concept to define precisely. This paper investigates the structural conception of the covalent bond, which says that bonding is a directional, sub-molecular region of electron density located between individual atomic centers that is responsible for holding the atoms together. Several approaches to constructing molecular models are considered in order to determine which features of the structural conception of bonding, if any, are robust across these models. The paper concludes that key components of the structural conception are absent in all but the simplest quantum mechanical models of molecular structure, seriously challenging the conception's viability.
\end{abstract}

Crucial to chemical practice and discourse is the notion of the chemical bond, specifically the covalent bond. For most synthetic and analytical purposes, molecules are conceived of not just as collections of atoms, but

*Earlier versions of this paper were presented at the Australia National University, the Philosophy of Science Association, and the Center for Philosophy of Science, University of Pittsburgh. I am grateful to these audiences for their enthusiasm and very helpful comments. Special thanks to Jerry Berson, Barry Carpenter, Dave Chalmers, Peter Godfrey-Smith, Clark Glymour, Robin Hendry, Roald Hoffmann, Paul Needham, John Norton, Janet Stemwedel, and Deena Skolnick Weisberg for extremely helpful feedback. 
as structural entities consisting of directionally oriented atomic centers connected, in some way or other, by covalent bonds. Hydrogen gas is said to be composed of two hydrogen atoms held together by a single, covalent bond; oxygen gas, two oxygen atoms and a double bond; methane, four equivalent $\mathrm{C}-\mathrm{H}$ single bonds.

While the chemical bond plays a central role in chemical predictions, interventions, and explanations, it is a difficult concept to define precisely. Fundamental disagreements exist between classical and quantum mechanical conceptions of the chemical bond, and even between different quantum mechanical models. Once one moves beyond introductory textbooks to advanced treatments, one finds many theoretical approaches to bonding, but few if any definitions or direct characterizations of the bond itself. While some might attribute this lack of definitional clarity to common background knowledge shared among all chemists, I believe this reflects uncertainty or maybe even ambivalence about the status of the chemical bond itself.

This kind of ambiguity has led chemists to different conclusions. Most commonly, chemists adopt a pragmatic stance and simply demand that bonding theories be useful for making predictions and aiding in the synthesis of new molecules. The underlying ontological status of the bond holds little interest. Although useful to everyday chemical practice, this approach is deeply unsatisfying to the philosopher of chemistry because it leaves unanswered fundamental questions about the nature of the chemical bond.

Other chemists, including ones of deep realist commitment, draw a different and more skeptical conclusion from these facts. Robert Mulliken, a founder of quantum chemistry said, "I believe the chemical bond is not so simple as some people seem to think." (Coulson, 1960) Charles Coulson, another founder wrote that the chemical bond "is a figment of our imagination." (1952) These chemists believe that progress in quantum chemistry shows that there is something deeply flawed about the chemist's notion of the bond. While this degree of skepticism may be warranted in the end, it is 
a hard thing for most chemists to accept given the ubiquity and usefulness of the bond concept.

Whatever the ultimate verdict, entertaining Coulson and Mulliken's skepticism raises many deep philosophical questions about the nature of chemical bonds and molecular structure. These questions include: Are chemical bonds real? If so, should we think of them as entities? If bonds are not real, is the phenomenon of bonding real? If so, how should this phenomenon be characterized? What is the relationship between molecular structure and bonding?

These issues are complex and interrelated, and philosophers of chemistry are beginning to consider them. (Woody, 1998; Needham, forthcoming; Berson, forthcoming; Stemwedel, forthcoming; Hendry, forthcoming) Approaches to answering these questions necessarily involve the combination of philosophical analysis and argumentation with a close examination of chemical theory itself. The latter has proved especially difficult because the last 80 years have seen myriad theoretical models of varying complexity developed to account for molecular structure. Much of the diversity comes from different approximations and idealizations that are made in the course of developing such models. Different models say different things about the phenomenon of covalent bonding and no single model is obviously the correct one, making the task of philosophical analysis more difficult. The problem of unclear foundations for the chemical bond is thus compounded by the diversity of models of structure and bonding.

This paper addresses one of these issues: It asks what the myriad models of molecular structure collectively say about the nature of bonding. Specifically, it asks whether models of molecular structure endorse a structural conception of covalent bonds, of the kind implicit in much of chemical practice. To cope with the multiplicity of models, I will follow a modified form of robustness analysis, where one looks for the structure common to multiple, otherwise conflicting models. (Levins, 1966; Wimsatt, 1981; Weisberg, 2006; Weisberg \& Reisman, forthcoming) As in standard robustness analysis, 
I will examine multiple models of molecular structure, looking for features and predictions common to these models. Where this paper will differ from standard robustness analysis is that we are already in possession of an external calibration point for the predictions of each model: experimentally measurable quantities. Using this external standard, we can examine successively more predictively accurate model types, investigating the general features that these models have and which ones they lack. Approaching the problem in this way will let us determine which bonding features of molecular models are on the surest theoretical footing, the features which ought to guide our inquiry about the foundations of covalency.

\section{The Structural Conception of Bonding}

The chemical literature often describes covalent bonds in structural terms, employing what I will call, following Hendry (forthcoming), the structural conception of bonding. In subsequent sections, I will investigate whether this conception is robust and if not, which features of bonding are robust. In order to carry out this analysis, I will rely on a working definition for the structural conception of bonding. Characterized in purely functional terms, the structural conception of bonding says:

A covalent bond is a directional, submolecular relationship between individual atomic centers that is responsible for holding the atoms together.

As this initial definition is functional, it does not say what realizes the role of the bond. Defining the bond this way will be useful in historical studies because bonds were an important part of chemical theory before the discovery that electrons played a role in bonding.

In modern chemistry, it is completely uncontroversial to see electrons or electron density as what realizes the submolecular relationship. This leads to the following structural conception of bonding: 
A covalent bond is a directional, sub-molecular region of electron density located between individual atomic centers that is responsible for holding the atoms together.

This conception tells us three important things about the nature of covalent bonds. First, it distinguishes covalent bonds from ionic bonds with the directionality restriction. Ionic bonds are omni-directional electrostatic interactions between positively and negatively charged ions. Covalent bonds are regions of electron density that bind atoms together along particular trajectories. Second, this conception says that bonding is a sub-molecular phenomenon, confined to regions between the atoms. This eliminates the possibility that bonds are a molecule-wide phenomenon. Third, the region of electron density between the atomic centers has to hold these centers together, which I will interpret to mean that they are closer together then they would have been in the absence of the bond. In other words, bonds are a stabilizing force for the molecule, and this stabilization will manifest itself in the amount of energy required to separate the atomic centers. ${ }^{1}$ The next sections ask how robustly this conception of the chemical bond is represented in models of molecular structure.

\section{$2 \quad$ Valence Bond Models}

Valence bond models are the first type of quantum mechanical treatment of molecular structure I will discuss. These models can be thought of as "bringing together complete atoms and then allowing them to interact to form bonds." (Carroll, 1998, 24) In other words, covalent bonds are formed when two isolated atoms, each possessing a full complement of protons, neutrons, and electrons, are brought together and allowed to interact. The change in electron distribution and the resulting energetic stabilization from

\footnotetext{
${ }^{1}$ For further discussion about the structural conception as well as other conception of covalent bonding, see Hendry, forthcoming.
} 
this change is taken to be the bond. All of this has to be unpacked in some detail, but we need to begin by considering what a quantum mechanical description of a molecule looks like.

The quantum mechanical description of atoms and molecules are given as wavefunction of the form $\psi(x, y, z, t)$. Different molecular modeling techniques will generate somewhat different wavefunctions for the molecule in question because of the different approximations they employ $\left.\right|^{2}$ In all of the bonding models I will discuss, several simplifying assumptions will be made: First, I will only focus on the electrons and hence on the electronic wavefunctions $3^{3}$ Second, I will only examine time-independent wavefunctions, taking into account the spatial properties of the electronic wavefunctions, but not how they evolve in time. Third, I will only discuss models of molecular hydrogen $\left(\mathrm{H}_{2}\right)$, a two-proton, two-electron system. Because this strongly coupled system has a singlet state as its ground state, I will simply assume that the electrons have opposite spin. This will prevent us from having to consider the complexities of the triplet (same spin) two electron system, at least at the beginning of the discussion.

I will begin the robustness analysis with the simplest possible valence bond model of hydrogen, one that most closely embodies the structural conception of bonding.

\subsection{Simple Valence Bond Model}

The first model of $\mathrm{H}_{2}$ is constructed by multiplying together two wavefunctions corresponding to the electrons originally associated with two individual hydrogen atoms. The electrons are still "tied" to the nuclei that they came to the bond with.

\footnotetext{
${ }^{2}$ In recent times another effective quantum mechanical method has taken root in the theoretical chemistry community. This is density functional theory, which gives prime place to the electron density

${ }^{3}$ The assumption that the nuclear and electronic wavefunctions can be computed separately is called the Born-Oppenheimer approximation. (Born \& Oppenheimer, 1927)
} 
Let $a$ and $b$ denote electronic wavefunctions centered around two distinct hydrogen nuclei. They will each bring along an electron which we will designate as 1 and 2 . This simple model keeps the first electron (1) in the vicinity of the first hydrogen nucleus $(a)$ and the second electron (2) in the vicinity of the second nucleus $(b)$, but multiplies them together to represent the mixing of the wavefunctions upon bring the atoms together. Here $a(1)$ stands for the atomic wave function (1s in the simplest case) of electron 1, moving around atomic center $a$. Mathematically, this can be expressed as follows, where $c$ is a normalization parameter:

$$
\psi_{1}=c a(1) b(2)
$$

Using this wavefunction, the Hamiltonian for the molecule, and the Schrödinger equation, we can calculate the dissociation energy of the molecule described by this wavefunction. The dissociation energy is the energy required to pull the atoms apart, removing the interaction between them and hence destroying the covalent bond. Using this model, the dissociation energy is calculated to be $24 \mathrm{~kJ} / \mathrm{mol}$. We can also calculate the equilibrium distance between the atomic nuclei. This gives us what is usually called the bond length. In this model, the equilibrium bond length is calculated to be $90 \mathrm{pm}$.

We can check these predictions against experimental measurements of these properties. The simple valence bond model predicts $24 \mathrm{~kJ} / \mathrm{mol}$ and $90 \mathrm{pm}$, but experimental investigation has measured the bond length to be $74.1 \mathrm{pm}$ and the dissociation energy to be $458.0 \mathrm{~kJ} / \mathrm{mol}$. We are in the right ball park, but clearly there is much room to improve our model. The first improvement will be to loosen the spatial restriction of each electron.

\subsection{The Heitler-London Model}

A substantial improvement in accuracy can be made to our simple model of the H-H bond. The model was first proposed by Heiter and London (1927). 
It starts from the model discussed in 2.1 , but modifies it by making the following observation: The electrons are indistinguishable and hence it is arbitrary to assign electron 1 to atomic center $A$ and electron 2 to atomic center $B$. The model thus considers a compound wavefunction, generated from the one discussed in $\$ 2.1$ and the following:

$$
\psi_{2}=c a(2) b(1)
$$

This corresponds to the electrons "swapping places."

In quantum mechanics, wavefunctions can be built up by taking linear combinations of simpler wavefunctions. Thus our full wavefunction will be the linear combinations of (1) and (2). The linear combination corresponding to the singlet ground state is:

$$
\psi_{\mathrm{HL}}=c a(1) b(2)+c a(2) b(1)
$$

Confining our attention then to (3), let's consider what the wavefunction tells us qualitatively about the distribution of electrons. If we think about them as particles, it tells us that they are free to move between the atoms, but in a coordinated fashion. They must on average be near opposite atomic centers, but they have greater mobility. It is impossible for a verbal description to be fully accurate, but a somewhat better description is that when we calculate the probability distribution of the electrons through space $\left(\Psi \Psi^{*}\right)$, we find that there is a buildup of electron density between the two atomic centers, with maximum electron density centered on each of the two atomic centers. The biggest difference between this model and the simpler one considered in the previous section is that there is a greater distribution of electron density throughout the molecule.

How well does the Heitler-London model predict the measurable properties of $\mathrm{H}-\mathrm{H}$ bonds? Considerably better than the simple valence bond model, it turns out. The bond distance is calculated to be $86.9 \mathrm{pm}$, which is a lit- 
tle better than the simple model, but the dissociation energy is calculated to be $303 \mathrm{~kJ} / \mathrm{mol}$, which now brings it to the same order of magnitude as the measured value. By allowing greater electron delocalization, the model is brought in to closer alignment with the experimentally measured values. This is a theme that I shall return to throughout the paper.

\subsection{Improving Valence-Bond Models}

The Heitler-London model can be improved further if we take the HeitlerLondon wavefunction to be just two terms of a more complex wavefunction. In other words, we add additional terms corresponding to other characteristics the wavefunction might have. I will only discuss one example of this, but it illustrates a very general principle at the heart of modern model building in quantum chemistry.

One thing that the Heitler-London model does not do is take in to account the possibility that the molecule could have ionic character. In other words, it does not allow for the possibility that there is a greater distribution of electron density on one side of the molecule or the other, however briefly or however slightly.

We can take this possibility in to account by adding in additional terms corresponding to an ionic distribution of electron density, which at the extreme would correspond to the electrons both being on one side of the molecule or on the other side (e.g. $a(1) b(1))$. At the same time, we do not want to over-compensate, adding too much ionic character. One way around this is to add an adjustable parameter $\lambda$ to each term. The wavefunction is then optimized by setting $\lambda$ to some value, calculating the energy of molecule, then trying another value for $\lambda$, and keep iterating until we find the value that gives the lowest energy, making the well-motivated assumption that the ground state electronic wavefunction will be the one with the lowest energy $\stackrel{4}{4}^{4}$ The new model has the following wavefunction:

\footnotetext{
${ }^{4}$ Technically, we also rely on the variation theorem which tells us that this procedure
} 


$$
\psi_{\text {ionic }}=c a(1) b(2)+c a(2) b(1)+\lambda c a(1) b(1)+\lambda c a(2) b(2)
$$

Adding these ionic terms considerably improves the model, allowing us to calculate $388 \mathrm{~kJ} / \mathrm{mol}$ and $74.9 \mathrm{pm}$, bringing the dissociation energy of the model even closer to the experimental value.

Having looked at three successively improved valence bond models, let's consider what they have told us collectively about the structural conception of bonding. First, by letting the electrons delocalize, as in the Heitler-London approach, the model came closer to agreement with experimentally measured quantities. When the model was made even more flexible, allowing the electrons to travel further afield and giving the model ionic character, the model came in even better agreement with experimental measurements. This trend continues with increasing electron mobility: In 1968, Kolos and Wolniewicz used a 100-term, highly flexible wavefunction, and after optimizing the parameters, they came within $0.01 \mathrm{~kJ} / \mathrm{mol}$ of the measured values. (Kolols \& Wolniewicz, 1968) Thus, the result most strongly robust among these many models of the covalent bond is that greater electron delocalization leads to greater stabilization and closer agreement to experiment.

What do these valence bond models say about our three criteria for the structural conception of bonds? First, they are directional and allow us to distinguish between ionic and covalent bonds. However, as we have just seen, adding ionic character actually stabilizes many molecular models such those for $\mathrm{H}_{2}$ model. Second, while the simple valence bond models start out with sub-molecular regions of electron density that we can identify as the bond, they are improved by allowing the electrons to have increased delocalization, spreading some of the density throughout the molecule. Finally, the valence bond models do nothing to challenge the idea that bonds hold the molecule

will only every overestimate the energy, but not under estimate. Thus we can keep varying parameters until we get the energy minimum, without fear that we are underestimating. (Levine, 1991) 
together.

\section{Molecular Orbital Models}

Our robustness analysis will continue with a second, distinct approach to constructing molecular models: the molecular orbital method. Rather than looking at bonding as what happens when we bring together two atoms and let them interact, we start off with atomic centers at initial distances, then calculate electron wavefunctions for the entire molecule. The electrons are affected by a potential field generated by the nuclei collectively. This is repeated at different nuclear distances, building up a potential energy curve, from which the equilibrium bond distance can be calculated.

As with valence bond models, one can start with an arbitrary form for the molecular orbital wavefunction, and then improve the model by adding more terms and adding adjustable parameters on these terms. We will start with a simple trial wavefunction for $\mathrm{H}_{2}$ to illustrate some of the key features of molecular orbitals. A prudent way to begin is to use wavefunctions corresponding to linear combinations of the atomic orbitals of our two hydrogen atoms, which we will call $A$ and $B$. So we have:

$$
\begin{gathered}
\psi_{\sigma_{g}}=\psi_{A}+\psi_{B} \\
\psi_{\sigma_{u}}=\psi_{A}-\psi_{B}
\end{gathered}
$$

When populated with electrons, equation 5 gives us the lower energy bonding wavefunction, while equation 6 gives us the higher energy antibonding wavefunction. If we want to calculate the total electronic wavefunction for the molecule, corresponding to adding the two electrons into the hydrogen molecular orbital, we take the product of the wavefunction for each 
orbital 5

$$
\psi_{H_{2}}=\psi_{\sigma_{g}(1)} \psi_{\sigma_{g}(2)}
$$

Combining equation 5 with equation 7 , we get the following expansions:

$$
\begin{gathered}
\psi_{H_{2}}=\left[\psi_{A(1)}+\psi_{B(1)}\right]\left[\psi_{A(2)}+\psi_{B(2)}\right] \\
\psi_{H_{2}}=\psi_{A(1)} \psi_{A(2)}+\psi_{B(1)} \psi_{B(2)}+\psi_{A(1)} \psi_{B(2)}+\psi_{A(2)} \psi_{B(1)}
\end{gathered}
$$

Interesting structure emerges from the terms in this equation. The third and fourth terms $\left(\psi_{A(1)} \psi_{B(2)}\right.$ and $\left.\psi_{A(2)} \psi_{B(1)}\right)$ are exactly the same terms as those found in the Heitler-London model of the $\mathrm{H}_{2}$ molecule. This corresponds to a single electron being located in the vicinity of each nucleus. However, there are two additional terms $\psi_{A(1)} \psi_{A(2)}$ and $\psi_{B(1)} \psi_{B(2)}$ which correspond to the electrons simultaneously positioned near the $A$ nucleus or the $B$ nucleus. These ionic terms corresponding to asymmetric electronic distributions had to be added manually to the Heitler-London model. So right from the start, this model includes the possibility of ionic character in what would classically be treated as a non-polar, covalent bond. In fact, in this molecular orbital wavefunction the covalent and ionic parts of the wavefunction are given exactly equal weight. We will come back to this point below.

How does this simple molecular orbital model conform to experiment? If we calculate the dissociation energy and bond distance predicted by this model, we come up with $-260 \mathrm{~kJ} / \mathrm{mol}$ and $85 \mathrm{pm}$ respectively, values which accord reasonably well with the measured values of $-458.0 \mathrm{~kJ} / \mathrm{mol}$ and $74.1 \mathrm{pm}$. These values are similar to those calculated with the Heitler-London model, but considerably better than those calculated with the simplest valence bond

\footnotetext{
${ }^{5}$ It is assumed (and suppressed for clarity) that this spatial wavefunction is multiplied by an antisymmetric spin wave function to describe the ground state of the molecule.
} 
Figure 1: A cross-section of the electronic density distribution calculated from a molecular orbital model of hydrogen. This diagram is not to scale.

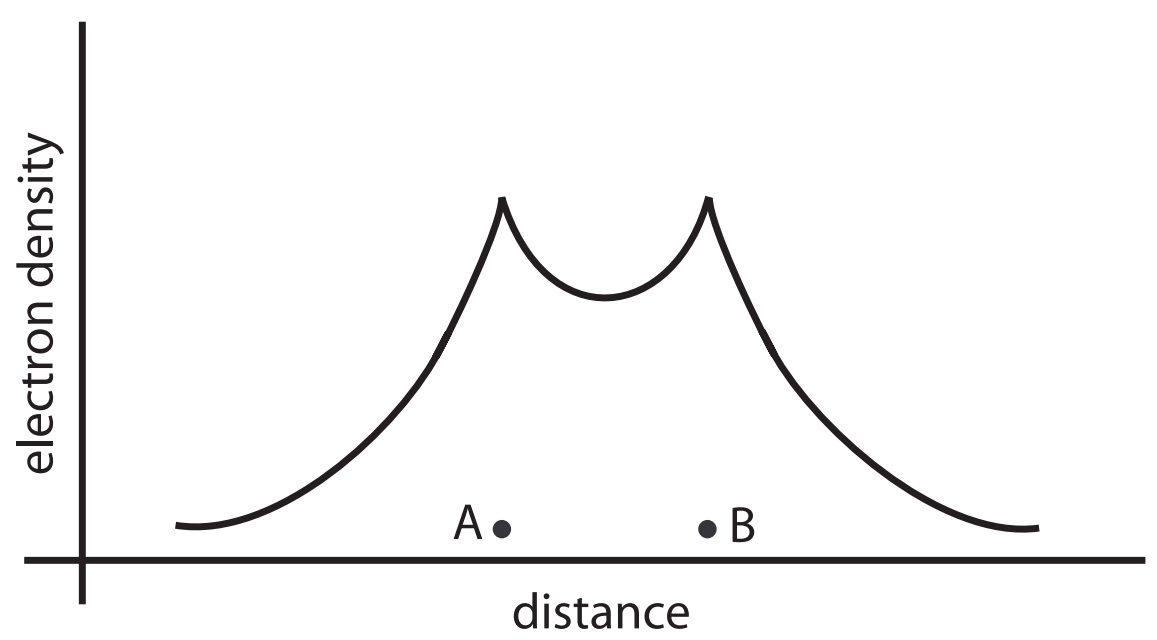

model. Even in this simple model, we see some interesting and non-classical structural features.

First of all, there is the importance of the aforementioned ionic terms. This suggests that there is far greater electron mobility than in a case of two isolated atoms. Further, the electronic density is at its peak in the areas immediately surrounding the nuclear centers. Figure 1 is a cross-section of the amplitude of the wavefunction of $\mathrm{H}_{2}$ calculated from our model. We can see that the highest density is just around the nuclear centers, with considerable density in between the atomic centers, but also a reasonable amount of electronic density on the far sides of the atomic centers.

There are many ways to improve this simple molecular orbital model, some of which will be discussed in the next section. But even from this very simple model and the Heitler-London model discussed in the last section, we can see the continuation of the pattern that has been emerging. Delocal- 
ization of electron density is a prominent feature of this model of molecular structure. The electrons are not sitting between the two atoms holding everything together; rather, they are distributed throughout the molecule. This puts pressure on the second part of the structural conception of bonds, the idea that bonds are sub-molecular phenomena. Similarly, delocalization makes the bonds less directional. The nuclei are still located in particular places that minimize the total energy of the molecule; however, the bonds themselves are considerably more diffuse. As we will see in the next section, the trend continues with more complex molecular orbital models.

\section{Modern Molecular Orbital Models}

The methods considered so far were worked out for simple molecules in the first half of the twentieth century. Since that time, there has been considerable progress in developing models which can yield more accurate calculations of molecular properties. To review all of these methods here would be impossible and unnecessary; however, many modern methods can be seen as systematic improvements to molecular orbital models along two dimensions relevant to the structural conception of bonds.

The first type of improvement involves using a hierarchy of more complex basis functions as trial wavefunctions. These basis functions are expanded by taking in to account many possibilities (with adjustable parameters), including greater delocalization of the electrons as well as occupation of higher energy electronic states. Just as ionic terms are "mixed" in to the valence bond wavefunctions, so too these spatially diffuse, delocalized, and higher energy states are mixed in to the starting molecular wavefunctions.

The second dimension on which modern methods improve upon simple molecular orbital models is in the nature of the optimization of the wavefunction, given the basis set. I have already alluded to the way calculations are typically performed using simple molecular models, a method called the 
Hartree-Fock method. The method works as follows:

1. Make an initial guess of the position of the nuclei.

2. Start with initial values of the parameters on all the electronic terms in the trial wavefunction.

3. Choose the terms corresponding to a single electron, then vary the parmaters on those terms in order to minimize the energy.

4. Repeat the step 3 for each electron in sequence.

5. Repeat step 4, cycling through all of the electrons until the energy values no longer change, having reached self-consistency of the orbitals with the field generated by the electrons.

6. Perturb the nuclei a small distance and repeat steps 2-5, searching for an energy minimum. (Levine, 1991; Ansyln \& Dougherty, 2005)

Although wavefunctions calculated with the Hatree-Fock method can yield excellent results, the method does not fully take in to account electron correlation, the coordinated movement of electrons. Recall that electronic wavefunctions are optimized from the point of view of a single electron. The method imagines that each electron is influenced by a field generated by the stationary distribution of all of the other electrons. It moves systematically through the electrons, treating them in this fashion, until self consistency is achieved. But any two electrons in the vicinity of one another will repel one another and this cannot be fully taken in to account, especially for electrons of anti-parallel spin, in this type of calculation. Hence a more accurate calculation would take this electronic correlation in to account. One method for doing this is called configuration interaction.

Configuration interaction approximates the effect of electron correlation by constructing a new molecular wavefunction from linear combinations of the ground state configuration (the one discussed throughout this paper) 
and wavefunctions that correspond to promoting electrons to higher-lying molecular orbitals, formally approximations to what might be the excited state of the molecule. Each of these terms has a coefficient that can be varied in order to minimize the total energy. The resultant wavefunction from a configuration interaction calculation will take the following form:

$$
\Psi_{C I}=c_{1} \Psi_{1}+c_{2} \Psi_{2}+c_{3} \Psi_{3}+\ldots
$$

A full conguration interaction calculation would take in to account all the possible ways that electrons can be assigned to the molecular orbitals formed from the basis set. In practice, only a few excited states are usually taken in to account because of the great computational cost of these calculations.

These approximation methods and others are interesting, but take us away from the important question that is at the heart of our robustness analysis: What do successively better calculations that take in to account CI tell us about bonds?

We can answer this question by reflecting on why configuration interaction allows us to build very good molecular models that take into account electron correlation. Remember that correlation is the property of the electrons moving out of the way of one another. Conceptually, adding in these repulsions will change the shape of the molecular orbital, because the electrons may spread apart further in space. A systematic way to alter the shape of molecular orbitals is to mix in orbitals corresponding to formal excited states, which will have different shapes and symmetry properties from the ground state orbital. When enough of these are mixed together, we will have the flexibility to accurately describe the real distribution of electrons in the molecule, through orbitals which correctly account for correlation as well as the nucleus-electron attractions that account for the electronic distribution. (Ansyln \& Dougherty, 2005, 824)

Configuration interaction is another example of how moving electrons out of the classical bonding region stabilizes the molecular model and brings it 
in to closer agreement with experimentally determined values.$^{6}$ When we allow for greater delocalization, taking in to account electrons temporarily entering higher energy states so as to avoid one another, molecular models increasingly agree with experimentally measurable quantities.

\section{Robust Properties of Chemical Bonds}

We have now come to the conclusion of our whirlwind tour of molecular models, searching for the robust properties of chemical bonds. In this concluding section, I will review what we have discovered, examining both what is and what is not robust across models of molecular structure.

Our examination of molecular models began with the simple valence bond model, the one that most faithfully illustrates the structural conception of bonding. In this model, electrons were associated with particular nuclei and as a result, the molecule was represented by bringing together two atoms, letting their electrons interact, and taking advantage of the stabilization created by this interaction. The electrons remained associated with a particular nucleus; their interactions were then described as sharing of the electrons between two atoms to form a bond.

This idea of electrons coming along with nuclei and being shared between two nuclei is resolutely not robust across bonding models. As we move in the direction of models which correspond more accurately to experimentally measurable values, this property diminishes and seems to vanish altogether in modern models.

The structural conception does not demand that electrons are shared or even fully located in the region between the atoms. Indeed it only demands that bonds can regularly be identified as directional, sub-molecular regions of electron density. So what we really need to know is whether such regions

\footnotetext{
${ }^{6} \mathrm{In}$ addition, CI also takes in to account the possibility that when one electron is in between the nuclei, the other one can be on the outside of the two nuclei.
} 
of density can be identified in molecular models. Answering this question is considerably more difficult and this paper certainly gives no definitive answer to it; however, the properties which we have found to be robust goes a long way towards answering it.

Among the bonding models, several things have been shown to be robust. In the examples we have considered, the models became energetically stabilized by forming the bond. Energy is liberated when the bond is formed - conversely, it takes energy to break a bond. This means that for atoms that readily form covalent bonds, it is energetically more stable for them to be in the bonding state than separated from each other such that the electrons cannot interact. Bonding seems to fundamentally involve energetic stabilization.

The second robust feature of the models is that greater electron delocalization leads to stabilzation. When we moved from the simple valence bond model to the Heitler-London model, we saw considerable stabilization. This accompanied relaxing the restriction that pinned electron 1 to nucleus $A$ and electron 2 to nucleus $B$. We further improved that model by adding terms to the basis function which allowed greater flexibility to the resultant wavefunction, which has the physical significance of allowing greater delocalization. In addition, the wavefunction is improved as we added correlation, the lowering of energy due to electrons being allowed to stay away from each other (as they remain attracted to the nuclei).

The same is true in the molecular orbital models. Even the simple molecular orbital models represent the electrons as delocalized across the molecule. Improvements to the molecular orbital model involved using basis functions with greater numbers of terms, which also allows more flexibility in describing the electronic distribution, a distribution which is not tied to the classical positioning of the electrons in between the nuclei.

These robust features of molecular models put considerable pressure on the structural conception of bonds, and are no doubt the sorts of results 
that led Coulson and Mulliken to their skepticism about the reality of bonds. To be explicit, the structural conception says that covalent bonds are directional, sub-molecular regions of electron density that hold molecules together. Throughout this paper, we have seen that delocalization - density spread beyond the sub-molecular region between the atoms - is the norm in molecular models. Further, the addition of ionic character, correlation, and the mixing of higher energy states all seem to improve the model, but make the bonds less directional and less sub-molecular.

I believe that this clearly rules out any straightforward version of the structural conception of covalent bonding. However, while the features I have pointed to that improve bonding models including correlation and ionic character involve deviations from local, sub-molecular regions of electron density, they themselves describe electronic characteristics of molecules in structural terms. They may in fact be compatible with a revised structural conception of bonding. While future analysis is required to either vindicate or fully reject the structural conception of bonding, the considerations in this paper go a long way towards motivating Coulson and Mulliken's skepticism about the reality of the covalent bond.

\section{References}

Ansyln, E. V., \& Dougherty, D. A. (2005). Modern physical organic chemistry. Sausalito, CA: University Science.

Berson, J. A. (forthcoming). Molecules with very weak bonds: The edge of covalency. Philosophy of Science.

Born, M., \& Oppenheimer, R. (1927). Zur Quantentheorie der Molekeln. Annalen der Physik, 74, 457-485.

Carroll, F. A. (1998). Perspectives on structure and mechanism in organic chemistry. Pacific Grove: Brooks/Cole. 
Coulson, C. A. (1952). What is a chemical bond? Scientific Journal of the Royal College of Science, 21, 11-29.

Coulson, C. A. (1960). Present state of molecular structure calculations. Reviews of Modern Physics, 32, 170-177.

Heitler, W., \& London, F. (1927). Wechselwirkung neutraler Atome und homöopolare Bindung nach der Quantenmechanik. Zeitschrift für Physik, 44, 455-472.

Hendry, R. (forthcoming). Two concepts of chemical bond. Philosophy of Science.

Kolols, W., \& Wolniewicz, L. (1968). Improved theoretical ground-state energy of the hydrogen molecule. Journal of Chemical Physics, 49, 404-410.

Levine, I. N. (1991). Quantum chemistry (4th ed.). Englewood Cliffs, NJ: Prentice Hall.

Levins, R. (1966). The strategy of model building in population biology. In E. Sober (Ed.), Conceptual issues in evolutionary biology (First ed., pp. 18-27). Cambridge, MA: MIT Press.

Needham, P. (forthcoming). Resisting chemical atomism: Duhem's argument. Philosophy of Science.

Stemwedel, J. (forthcoming). Chemical bonds in chemical explanations. Philosophy of Science.

Weisberg, M. (2006). Robustness analysis. Philosophy of Science, 73.

Weisberg, M., \& Reisman, K. (forthcoming). The robust volterra principle. Philosophy of Science.

Wimsatt, W. C. (1981). Robustness, reliability, and overdetermination. In M. Brewer \& B. Collins (Eds.), Scientific inquiry and the social sciences (pp. 124-163). San Francisco: Jossey-Bass.

Woody, A. (1998). Early twentieth century theories of chemical bonding: Explanation, representation, and theory development. Unpublished doctoral dissertation, University of Pittsburgh. 